\title{
La utilidad del procedimiento del Milieu Teaching Mejorado en bebés con severos problemas de comunicación y lenguaje en el contexto clínico
}

\author{
Marina Calleja, José Miguel Rodríguez y María Luisa Luque \\ Universidad de Málaga, España
}

\begin{abstract}
Resumen
En el presente trabajo se muestran los resultados de dos niños de corta edad con severos problemas de comunicación y del lenguaje a los que se les aplicó una metodología de intervención de corte naturalista denominada Enhanced Milieu Teaching Procedure (EMTP) en unas condiciones especiales: a) los bebés estaban fuera de las condiciones óptimas para aplicar el método y; b) se introdujo una fase híbrida de entrenamiento con el logopeda al final del proceso. Las condiciones óptimas para aplicar el EMTP según Hancock \& Kaiser (2009) es cuando los niños sean capaces de realizar imitación verbal, tengan un repertorio comunicativo de al menos 10 palabras y, con una longitud media de enunciado de entre 1 y 3,5. Tras la aplicación de EMTP durante 6 meses, los bebés mostraron un incremento en su repertorio comunicativo, extrapolando lo aprendido a otros ambientes (escolares y familiares). Incluso con limitaciones severas, cuando aún no se disponen de los prerrequisitos propuestos por los teóricos, el EMTP es efectivo.

Palabras clave: Intervención naturalista; Milieu teaching; Habilidades comunicativas.
\end{abstract}

\section{The usefulness of the Enhanced Milieu Teaching Procedure in toddlers with severe communication and language disorders in the clinical context}

\begin{abstract}
This study shows the results of a naturalistic intervention procedure called Enhanced Milieu Teaching Procedure (EMTP) as an intervention methodology in two toddlers with severe communication and language disorders. We use the intervention procedure within some specific and special conditions: a) toddlers didn't fulfill the optimal characteristics suggested for applying EMTP; b) we introduce a novel training stage at the end of the intervention procedure, which consisted in a speech and language therapist training the child. Hancock \& Kaiser (2009) suggested several optimal conditions for the success applying EMTP; The child must be verbally imitative, must have at least ten productive words and between 1-3,5 mean length of utterance. The two toddlers showed an increasing progress in their communicative productions, moreover those productions became wide spread in naturals settings (home and school). The EMPT is effective even in toddlers with severe communication impairments and without the suited prerequisites suggested so far.

Key words: Communication skills; Milieu Teaching Procedure; Naturalistic intervention.
\end{abstract}

Correspondencia con los autores: marinac@uma.es

Recibido 11 de Julio. Primera revisión 1 de Octubre. Aceptado 3 de Diciembre de 2013 


\section{Introducción}

La efectividad de las estrategias tradicionales de entrenamiento de ensayos discretos ha quedado constatada en estudios de corte experimental (e.g. Sheinkopf \& Siegel, 1998; Smith, Groen \& Wynn, 2000), sin embargo para algunos profesionales dichos métodos no resultan excesivamente apropiados cuando los niños presentan severos déficits de comunicación a causa de su limitada generalización de las nuevas habilidades aprendidas a entornos cotidianos (Cowan \& Allen, 2007).

Frente a los métodos tradicionales de intervención logopédica (para una revisión de estos métodos ver Carr, 1985; Koegel, O'Dell \& Koegel, 1987), la interacción niñoadulto en entornos naturales ha proporcionado excelentes oportunidades para el desarrollo del lenguaje infantil, por el peso que se le concede al entorno social en la adquisición del lenguaje (Harjusola-Webb \& Robbins, 2012; Vilaseca \& del Río, 1997).

Entre los modelos de intervención de corte naturalista se encuentra el Milieu Teaching Procedures (MTP o enseñanza ambiental). El MTP es un modelo de intervención logopédica instruccional cuya finalidad es abordar los déficits comunicativos en niños con dificultades cuando el entorno no proporciona la ayuda necesaria para superar dicho déficit (Alper \& Kaiser, 1992). Este procedimiento fue planteado por primera vez por Hart y Rogers-Warren en 1978 como un modelo general de intervención en el ámbito de la comunicación y el lenguaje en el que las sesiones de intervención no estaban estructuradas. El punto de partida era la iniciativa atencional del niño, a través de la cual se pretendía enseñar la forma y el contenido del lenguaje dentro de un contexto habitual de intercambios conversacionales (v.g. el juego).

En la última década se ha reformulado el MTP y se está aplicando el modelo de Enseñanza Ambiental Mejorado (en inglés Enhanced Milieu Teaching Procedure o EMTP -sus siglas en inglés-). Esta versión es un modelo de intervención híbrido que combina estrategias procedentes del modelo interactivo (Girolametto, Weitzman \& Greenberg, 2003; Kaiser, Hemmeter \& Hester, 1996) ${ }^{1}$, y los enfoques de intervención naturalista (Alpert \& Kaiser, 1992; Hancock \& Kaiser, 1996). ${ }^{2}$

\footnotetext{
7 Este modelo se basa en la interacción receptiva (la retroalimentación contingente, el modelado de elementos-objetivo de lenguaje y las expansiones).

2 Este modelo pretende facilitar la producción del lenguaje mediante los modelos de elicitación, mandatos, demora temporal y enseñanza incidental (para una revisión detallada del método ver Hancock y Kaiser, 2009).
} 
Aunque este modelo procede del ámbito naturalista, determinados autores plantean la necesidad de incorporar al modelo la participación de los clínicos, a fin de incrementar la eficacia del proceso. Una característica adicional del presente estudio radica en la complementariedad de los contextos naturalistas y clínicos. La aplicación del EMTP se centra en estimular el uso funcional de las habilidades de lenguaje productivo en interacción en un entorno natural y, por tanto, los padres juegan un papel central para enseñar el lenguaje en este entorno a sus hijos. Sin embargo, algunos estudios sugieren que la intervención clínica no naturalista es necesaria no solo para entrenar con eficacia a los padres sino también para aplicar las estrategias EMTP a los propios niños (Hancock \& Kaiser, 2009). Además, el EMTP implica una creciente flexibilidad para modelar y facilitar la imitación del niño, lo cual incluye una amplia variedad de actividades de juego, contextos de entrenamiento y conductas contingentes que tienen que poner en marcha tanto el clínico como el niño (Camarata, 2000). Por todo ello, la forma de maximizar la eficacia del EMTP es cuando se interviene con padres entrenados y con clínicos (Gilbert, 2008).

A continuación exponemos una breve explicación de las estrategias EMTP para garantizar la comprensión del estudio empírico que hemos realizado (para un análisis más detallado ver Choi \& Kim, 2005; Christensen-Sandfort \& Whinnery, 2013). Las estrategias que incorpora el EMTP son:

a) La interacción receptiva incluye un conjunto de conductas como son: seguir las iniciativas del niño, responder a las iniciaciones verbales y no verbales del niño, proporcionar retroalimentación contingente o consecuencias positivas así como las expansiones de los enunciados del niño, lo cual mantiene el interés del niño en la conversación y proporciona un modelo lingüístico para mejorar el lenguaje habitual del mismo.

b) El modelado se emplea en la fase inicial. Consiste en mostrar al niño la respuesta que estamos buscando. Las respuestas lingüísticas modeladas tienen la peculiaridad de que o bien no se encuentran entre el repertorio comunicativo del niño o le resultan muy complicadas de poner en práctica.

c) El modelo mandato consiste en hacer al niño la siguiente pregunta “¿qué quieres?” o "dime qué quieres". La finalidad es que el niño genere la respuesta específica en relación con sus intereses. Esta estrategia se emplea una vez que el niño es capaz de 
imitar la respuesta lingüística deseada aunque tenga lagunas en las habilidades conversacionales.

d) El retraso temporal consiste en esperar durante un corto periodo de tiempo tras establecer la atención compartida, a fin de dar pie a la conducta comunicativa esperada o deseada por parte del niño.

e) La enseñanza incidental es la estrategia que ampara a las otras estrategias anteriores (modelado, modelo mandato y retraso temporal). Con esta estrategia los adultos estructuran cuidadosamente el entorno para fomentar las conductas comunicativas (peticiones, comentarios, etc...) del niño.

Además de las estrategias indicadas, el método cuenta con una secuencia organizativa que incluye tres componentes: 1) organizar el ambiente para apoyar la enseñanza y aprendizaje del lenguaje (es decir, elegir actividades interesantes para el niño a partir de su actividad espontánea), 2) organizar las oportunidades naturales para facilitar el lenguaje y, 3) proporcionar las consecuencias positivas naturales de utilizar el lenguaje (Hancock \& Kaiser, 2009). Así, el conjunto de técnicas que conforman EMTP pretende dotar a los niños de las oportunidades necesarias para poner en práctica las destrezas comunicativas durante las interacciones entre un adulto y un niño, que suelen darse de forma espontánea en una situación desestructurada, como es la del juego libre. Las intervenciones infantiles son usadas de manera sistemática por los adultos para transmitir nueva información o propiciar el desarrollo de la habilidad comunicativa. El niño controla las incidencias en las que la enseñanza ocurre, al focalizar su interés en un aspecto concreto del ambiente. No es raro que los niños inicien la interacción solicitando la asistencia de los adultos. El elemento que diferencia EMTP de otros enfoques centrados en el niño es el uso de una secuencia de estímulos cada vez más específicos, a fin de asegurar la adquisición y uso por parte de los niños de los elementos-objetivo de la intervención (Hancock \& Kaiser, 1996).

Aunque el EMTP ha mostrado un incremento en las habilidades comunicativas de jóvenes con retraso en el lenguaje (Hancock \& Kaiser, 2002), incluyendo también a niños autistas (Hancock \& Kaiser, 2002, Kaiser, Hancock \& Neitfeld, 2000), niños con trastorno específico del lenguaje (Warren, McQuarter \& Rogers Warren, 1984) y niños con discapacidad severa (Halle, Marshall \& Spradlin, 1979; Kaiser, 1993, Kaiser \& Delaney, 2001), es más probable que muestre mejores resultados cuando los niños 
cumplan los siguientes requisitos y así, puedan responder al modelado del adulto (Hancock, y Kaiser, 2009):

- Niños que realizan imitación verbal

- Niños que tienen un repertorio comunicativo de al menos 10 palabras

- Niños cuya longitud media de enunciado está entre 1 y 3,5.

Todos los estudios, anteriormente citados, que utilizan el EMTP se han ceñido a las condiciones de uso planteadas por el método. No tenemos constancia de otros estudios que utilicen el EMTP en niños muy pequeños fuera de esas condiciones de uso. Esta circunstancia la consideramos una limitación dado que pensamos que este método tiene posibilidades de éxito en circunstancias comunicativas aún más restrictivas. En el presente trabajo se pretende demostrar que se puede utilizar con éxito el EMTP para incrementar el repertorio comunicativo aun cuando los niños se sitúen por debajo de los requisitos planteados (Hancock \& Kaiser, 2009), es decir, a) cuando no realicen imitación verbal alguna b) cuando su repertorio mínimo es inferior a 10 palabras, c) y cuando su longitud media de enunciados (LME) es inferior a 1'5.

En concreto, lo que se pretendió fue la adquisición e incremento de las primeras producciones verbales en dos niños de muy corta edad, con ausencia de producciones en la línea base, mediante la aplicación del EMTP en contexto natural entrenando a los padres y en contexto clínico. Las primeras producciones verbales se seleccionaron siguiendo el inventario de desarrollo comunicativo McArthur (López-Ornat, Gallego, Gallo, Karousou, Mariscal \& Martínez, 2005). Además, se tomó en cuenta las primeras 100 palabras con mayor frecuencia de uso entre 12-23 meses de edad (Serra i Raventós, Serrat, Soñé, Bel \& Aparici, 2000).

\section{Participantes}

Los niños que han participado en esta experiencia han sido dos bebés que fueron remitidos por las guarderías a las que acudían por mostrar ausencia total de lenguaje atendiendo a su edad y dificultades de interacción tanto con sus iguales y como con los adultos.

PL es una niña de 33 meses de edad acude por sospecha de autismo con estereotipias y ausencia de lenguaje. Los padres han visitado a diferentes profesionales 
y se han descartado problemas de visión, audición y alteraciones neurológicas. La niña utiliza los gestos, expresiones faciales y vocalizaciones preverbales para comunicarse.

FL es un niño de 25 meses de edad que presenta, entre otros síntomas, una producción verbal nula y ausencia de interés por sus iguales. Los padres descartaron problemas de visión, audición o alteraciones neurológicas tras acudir a diferentes profesionales médicos. El niño utilizaba gestos, expresiones faciales y vocalizaciones preverbales para comunicarse.

\section{Materiales}

Previamente a la aplicación del método, se procedió a una primera valoración de los recursos comunicativos y cognitivos de ambos bebés. Para conocer el nivel de desarrollo cognitivo se administraron las siguientes pruebas: la Escala de Desarrollo Psicomotor de la Primera Infancia Brunet-Lezine Revisada (BL-R), para conocer su edad y cociente de desarrollo (Josse, 1997) y, el Análisis del Juego Simbólico (JS) según las categorías de McCune-Nicolich (1995), para conocer sus habilidades de representación y simbolización. Para conocer su nivel de desarrollo comunicativo se utilizaron a) el Inventario de Comunicación (IC) de Manolson (1992) para identificar las destrezas comunicativas que utilizaban, d) Tarea de repetición de sílabas (RS) para valorar su conducta de imitación verbal y su repertorio fonológico. Los resultados se muestran en la Tabla 1.

\section{Método}

Se utilizó un diseño de caso único a partir de múltiples líneas base de los niños en dos actividades (Baer, Wolf \& Risley, 1968; Christensen-Sandfor \&Whinnery, 2013; Harjusola-Webb \& Robbins, 2012). Este diseño incluyó cuatro fases diferenciadas: Fase 1 o Fase de Línea Base; Fase 2 o Fase de Entrenamiento a padres; Fase 3 o Fase de intervención logopédica; y, Fase 4 o Fase de Mantenimiento (tras un mes de descanso sin entrenamiento con el logopeda).

La variable dependiente fue el número de producciones espontáneas de los elementos-objetivo comunicativos en las situaciones de juego estructurado y juego no estructurado y en los cuatro momentos marcados por el diseño. Las producciones espontáneas se definieron como los intentos comunicativos iniciados por el niño en ausencia de un modelo o modelo mandato dentro de una secuencia de instrucción. En la 
situación de juego estructurado, dicha secuencia incluía: a) la presentación de los materiales con o sin una ayuda; b) la producción por parte del niño de una respuesta correcta, una no respuesta o una respuesta incorrecta y, c) obtención de los materiales con retroalimentación contingente. En la situación de juego no estructurado, se trataba de utilizar el juego libre y espontáneo del niño a partir de sus propios intereses. Se seleccionaron elementos-objetivo comunicativos similares para ambos niños, utilizando los mismos materiales (juguetes y encajables).

Tabla 1: Datos valoración inicial

PL

FL

\begin{tabular}{lcc}
\hline EC & 33 meses & 25 meses \\
\hline BL-R & EE: 18:12 & EE: $22: 12$ \\
& C.D. 56 & C.D.92 \\
\hline JS & Uso de juguetes realistas, escasas & Uso de juguetes realistas, escasas \\
& $\begin{array}{c}\text { acciones sobre un agente pasivo, } \\
\text { secuencia ilógica de dos acciones, } \\
\text { preparación de acciones aisladas. }\end{array}$ & $\begin{array}{c}\text { acciones sobre un agente pasivo, } \\
\text { secuencia ilógica de dos acciones, } \\
\text { preparación de acciones aisladas. }\end{array}$ \\
\hline IC & Nivel II: & Nivel II: \\
& $\begin{array}{c}\text { Comunicación por contacto visual, } \\
\text { deícticos, expresiones faciales y } \\
\text { movimientos corporales }\end{array}$ & $\begin{array}{c}\text { Comunicación por contacto visual, } \\
\text { deícticos, expresiones faciales y } \\
\text { movimientos corporales }\end{array}$ \\
\hline RS & 0 & 0 \\
\hline $\begin{array}{l}\text { Producción } \\
\text { verbal }\end{array}$ & 0 & 0 \\
\hline $\begin{array}{l}\text { EC: edad cronológica en meses; BL-R: Escala de desarrollo Brunet-Lézine- Revisada; E.E.: edad equivalente; J.S.: Juego simbólico; } \\
\text { IC; inventario de comunicación; RS: repetición de sílabas. }\end{array}$
\end{tabular}

Los elementos-objetivo comunicativos, se concretaron siguiendo las directrices del inventario de desarrollo comunicativo de MacArthur para las primeras producciones (8-15 meses) (López-Ornat et al., 2005) y las 100 primeras palabras con mayor frecuencia de uso (Serra i Raventós et al., 2000), quedando las siguientes agrupaciones: a) Sustantivos: sustantivos de diferentes categorías semánticas (juguetes, partes del cuerpo, animales, alimentos, ropa y objetos de la casa de uso frecuente).

b) Acciones: verbos relacionados principalmente con su actividad cotidiana, fisiológicos y de acción.

c) Personas: nombres de los parientes próximos (mamá/mami, papá/papi, abuela/yaya, abuelo/yayo) y nombres propios de parientes cercanos (sólo en el caso del niño FL).

d) Juegos, rutinas y fórmulas sociales: se concretaron en afirmación y negación (si, no), 
saludo y despedida (hola, adiós) y rutinas en la estructuración de la terapia generalizables a su actividad cotidiana (ya está, se acabó, vamos, a jugar). Se incluyó la fórmula para la petición (dame).

e) Pronombres y determinantes: entre los que se eligieron los de uso más frecuentes: este-esta, otro-otra y se incluyó el locativo aquí (cfr. Serra i Raventós et al., 2000).

Para operativizar los registros de frecuencia de producciones se distinguió entre categorías utilizadas como elementos comunicativos-objetivo de carácter abierto, cuyo número no se definió de antemano sino en función del interés del niño y la longitud de la palabra, y aquellas de carácter cerrado, definidas de antemano siguiendo las directrices señaladas. Los objetivos comunicativos de carácter abierto fueron sustantivos y acciones. Y los objetivos de carácter cerrado fueron el resto de las categorías: personas (formado por cuatro elementos), juegos, rutinas y fórmulas sociales (formado por nueve elementos) y pronombres determinantes y locativos (formado por cinco elementos). En ambos tipos de objetivos se registró el número total de producciones. Obsérvese que los objetivos comunicativos cerrados estaban limitados de antemano, sin embargo los abiertos no lo estaban y por ello han sido tratados en los resultados de forma diferencial.

A continuación se definieron las producciones espontáneas que iban a ser medidas. Dado que nuestros participantes tienen una edad cronológica inferior a los tres años y se encuentran en la fase de las primeras palabras, es esperable que sus producciones presenten una estructura de palabra reducida, estructura de sílaba con omisiones de coda y desarrollo fonológico incompleto con múltiples procesos de asimilación y sustitución de fonemas (véase los trabajos de Aguilar y Serra, 2003; Serra i Raventós et al., 2000). En nuestro estudio hemos considerado palabras aquellas producciones que cumplían los siguientes criterios: a) eran consistentes con la presencia o demanda del objeto o acción; b) tenían la intención de nombrar un referente en presencia o demanda del objeto o acción; c) conservaba la estructura de la palabra en relación al número de sílabas (la mayoría bisílabas y alguna trisílaba muy familiar) y la acentuación de la palabra. Todo ello, con independencia de otros procesos de simplificación (reducciones de sílabas, omisiones y sustituciones de fonemas) del habla infantil presentes en dichas producciones puesto que entre los dos y tres años, la edad de nuestros participantes, son habituales tanto el desarrollo fonológico incompleto (no han 
adquirido todos los fonemas) como la presencia de alteraciones en la estructura de palabra, sílaba y en los fonemas.

\section{Recogida de datos}

Fase 1 o de Línea Base

En esta fase se obtuvo la línea base a partir de las sesiones de evaluación inicial (un total de tres), en las que los niños participaron en actividades de juego estructurado y no estructurado. En esta fase se valoró el número de producciones y se clasificaron en los distintos elementos-objetivo comunicativos de los dos niños siguiendo las categorías descritas para las producciones espontáneas (Ver Tabla 2).

Tabla 2: Producciones durante la Línea Base (Fase 1)

\begin{tabular}{lcccc}
\hline & \multicolumn{2}{c}{ Juego Estructurado } & Juego No Estructurado \\
\hline Tipo de producción & PL & FL & PL & FL \\
\hline Sustantivos & 0 & 0 & 0 & 0 \\
Acciones & 0 & 0 & 0 & 0 \\
Personas & 0 & 0 & 0 & 0 \\
Juegos, rutinas y fórmulas sociales & 0 & 0 & 0 & 0 \\
Pronombres, determinantes y locativos & 0 & 0 & 0 & 0 \\
\hline
\end{tabular}

Fase 2 o Fase de entrenamiento a padres.

En esta fase se implementaron las estrategias EMTP en actividades estructuradas y no estructuradas con alguno de los progenitores. Por una parte, la actividad estructurada se organizó enseñando los materiales directamente. Mientras que en la actividad no estructurada, se dispusieron los materiales en un segundo plano, pero al alcance de la vista de los niños, junto con materiales para el juego libre. Todos los juguetes seleccionados estaban encaminados a la consecución de los elementos-objetivo comunicativos. La sesión tenía una duración de 40 minutos distribuidos en 15 minutos dedicados a actividades estructuradas, otros 15 minutos al juego libre (bien con juguetes propuestos por el terapeuta como por el niño) entre los que se intercalaban 10 minutos para atender las demandas de interés del niño, ajenas a la situación de juego y que pudieran producirse durante la sesión de entrenamiento.

La intervención tuvo lugar en contexto clínico acompañado de uno de los progenitores. Las sesiones de esta fase servían de entrenamiento a los padres en las estrategias EMTP con el fin de que éstos llegaran a adquirir algunas de las siguientes 
habilidades: a) reconocer los intentos comunicativos de su hijo y responder a su iniciativa, b) modelar y ampliar el juego y el lenguaje, c) aprender a poner en práctica la enseñanza incidental ${ }^{3}$, d) prestar atención a la disposición ambiental del juego y la organización los juguetes, e) familiarizarse con el uso de la demora de respuesta ${ }^{4}$, f) ejercitar en el reconocimiento y entrenamiento en el uso de los turnos de palabra y las expansiones verbales. Durante 15 sesiones los padres observan, participan y siguen las instrucciones de las técnicas descritas específicamente para reconocer e incrementar los intentos comunicativos ${ }^{5}$. En las dos últimas sesiones se registran las producciones espontáneas en ambas situaciones experimentales (juego estructurado y no estructurado) y se clasificaron por categorías siguiendo el procedimiento type-token. Los resultados se recogen en la tabla 3.

Tabla 3: Producciones durante la fase entrenamiento a padres (Fase 2)

\begin{tabular}{lcccc}
\hline & \multicolumn{3}{c}{ Juego Estructurado } & \multicolumn{2}{c}{ Juego No Estructurado } \\
Tipo de producción & PL & FL & PL & FL \\
\hline Sustantivos & 4 & 5 & 1 & 2 \\
Acciones & 1 & 3 & 1 & 1 \\
Nombres de personas & 0 & 0 & 0 & 0 \\
Juegos, rutinas y fórmulas sociales & 1 & 1 & 0 & 1 \\
Pronombres, demostrativos y locativos & 0 & 0 & 0 & 0 \\
\hline
\end{tabular}

\section{Fase 3 o Fase de Intervención logopédica:}

Siguiendo las indicaciones para el uso del método en contexto clínico, se introduce la fase 3, en la que la intervención logopédica se realizaba en ausencia de los padres, en el mismo contexto clínico, siguiendo los mismos procedimientos y número de sesiones que en la fase 2. En las dos últimas sesiones se registran las producciones espontáneas en ambas situaciones experimentales y se clasifican por categorías siguiendo el procedimiento type-token descrito para la Fase 2 (Los resultados se recogen en la Tabla 4).

\footnotetext{
3 Cuando el niño inicia la interacción y el adulto hace uso de esa oportunidad para requerir al niño una emisión más completa o elaborada. Al niño se le ayuda con estrategias del tipo de pregunta abierta o de alternativa forzada, imitación, petición de clarificación u otras (Warren \& Kaiser, 1986)

${ }^{4}$ Se trata de ofrecer ayuda al niño para que inicie la comunicación

5 Para una exposición más detallada del método de entrenamiento a padres ver http://kc.vanderbilt.edu/kidtalk/files/presentations/Kaiser_AUCD\%20Webinar_2_22_11.pdf.
} 
Tabla 4: Producciones durante la Intervención logopédica (Fase 3)

\begin{tabular}{|c|c|c|c|c|c|}
\hline \multirow[b]{2}{*}{ Tipo de producción } & & \multicolumn{2}{|c|}{$\begin{array}{c}\text { Juego } \\
\text { Estructurado }\end{array}$} & \multicolumn{2}{|c|}{$\begin{array}{c}\text { Juego No } \\
\text { Estructurado }\end{array}$} \\
\hline & & PL & FL & $\mathbf{P L}$ & FL \\
\hline Sustantivos & & 17 & 16 & 9 & 20 \\
\hline Acciones & & 6 & 14 & 5 & 10 \\
\hline Personas & & 3 & 4 & 1 & 4 \\
\hline Juegos, rutinas y fórmulas sociales & & 7 & 9 & 7 & 9 \\
\hline $\begin{array}{l}\text { Pronombres, demostrativos } \\
\text { locativos }\end{array}$ & $\mathrm{y}$ & 1 & 3 & 1 & 3 \\
\hline
\end{tabular}

Fase 4 o Fase de mantenimiento:

Tras un periodo de un mes sin intervención del logopeda en contexto clínico, se procedió a la medición de las producciones espontáneas en situación de juego estructurado y no estructurado en dicho contexto, a fin de llegar a establecer el grado de generalización y mantenimiento de los elementos-objetivo comunicativos-lingüísticos. En las tres sesiones de recogida de datos de la fase de mantenimiento, se registraron las producciones espontáneas y se clasificaron por categorías siguiendo el procedimiento type-token descrito para las Fases 2 y 3 (ver tabla 5).

Tabla 5: Producciones durante la Fase de mantenimiento (Fase 4)

\begin{tabular}{lcccc}
\hline & \multicolumn{3}{c}{ Juego Estructurado } & \multicolumn{2}{c}{ Juego No Estructurado } \\
Tipo de producción & PL & FL & PL & FL \\
\hline Sustantivos & 20 & 56 & 15 & 36 \\
Acciones & 12 & 34 & 9 & 20 \\
Personas & 4 & 5 & 4 & 5 \\
Juegos, rutinas y fórmulas sociales & 9 & 9 & 9 & 9 \\
Pronombres, demostrativos y locativos & 2 & 3 & 1 & 3 \\
\hline
\end{tabular}

\section{Resultados}

Para el análisis de los resultados vamos a partir de una tabla de datos que resume y agrupa las diferentes aportaciones presentadas en las diferentes fases del método (Ver Tabla 6). 


\begin{tabular}{|c|c|c|c|c|c|c|c|c|}
\hline \multirow[b]{3}{*}{ Fase } & \multicolumn{4}{|c|}{ FL } & \multicolumn{4}{|c|}{ PL } \\
\hline & \multicolumn{2}{|c|}{ JNE } & \multicolumn{2}{|c|}{ JE } & \multicolumn{2}{|c|}{ JNE } & \multicolumn{2}{|c|}{$\mathrm{JE}$} \\
\hline & $\mathrm{CA}$ & $\mathrm{CC}$ & $\mathrm{CA}$ & $\mathrm{CC}$ & $\mathrm{CA}$ & $\mathrm{CC}$ & $\mathrm{CA}$ & $\mathrm{CC}$ \\
\hline LB & 0 & 0 & 0 & 0 & 0 & 0 & 0 & 0 \\
\hline EP & 3 & 1 & 8 & 5 & 2 & 0 & 1 & 1 \\
\hline IL & 30 & 16 & 30 & 16 & 14 & 9 & 23 & 11 \\
\hline $\mathrm{MnT}$ & 56 & 17 & 90 & 15 & 27 & 14 & 32 & 13 \\
\hline
\end{tabular}

LB: Linea Base; EP: Entrenamiento a Padres; IL: Intervención Logopédica; MnT: Mantenimiento; CA: Clase Abierta; CC: Clase Cerrada.

El primer análisis que vamos a realizar pretende mostrar la evolución global de ambos niños a lo largo de todas las sesiones de trabajo, lo que podemos considerar el patrón de evolución (Ver Figura 1). En este análisis sólo hemos tenido en cuenta las producciones globales de ambos sujetos, sin distinción de categorías.

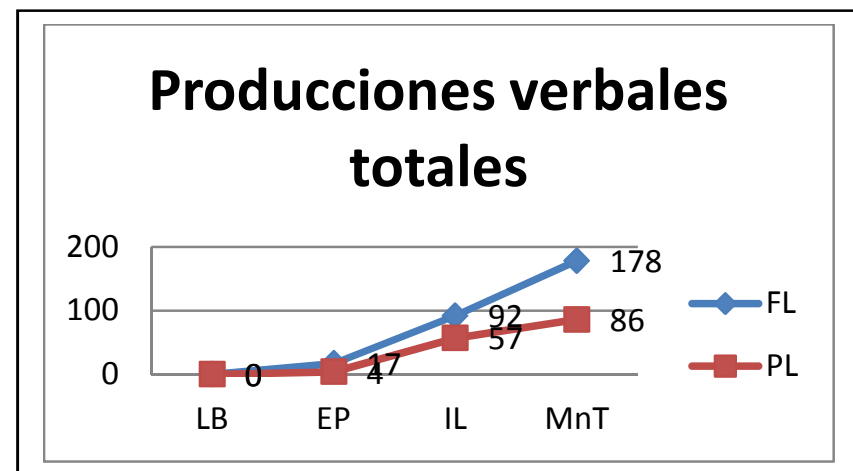

Figura 1 Evolución global de los dos niños

Lo hemos considerado una medida global. Atendiendo a los resultados, podemos observar lo siguiente:

En primer lugar, podemos constatar que las producciones de ambos niños se han incrementado a lo largo de todo el proceso de intervención, pasando de una producción global en línea base de cero (0) elementos a una producción final, medida en la fase de mantenimiento, de 178 elementos para FL y de 86 elementos para PL. Teniendo en cuenta sólo la globalidad de los datos, hemos de considerar que el método ha sido eficaz, aunque no lo haya sido por igual para cada uno de los niños.

En segundo lugar, hemos presentado los datos distinguiendo entre la condición 
de juego estructurado (JE) y Condición de juego no estructurado (JNE). Como era de esperar, en la situación de juego estructurado se observa un rendimiento mayor en ambos niños frente a la situación de juego no estructurado (Ver Figuras 2 y 3).

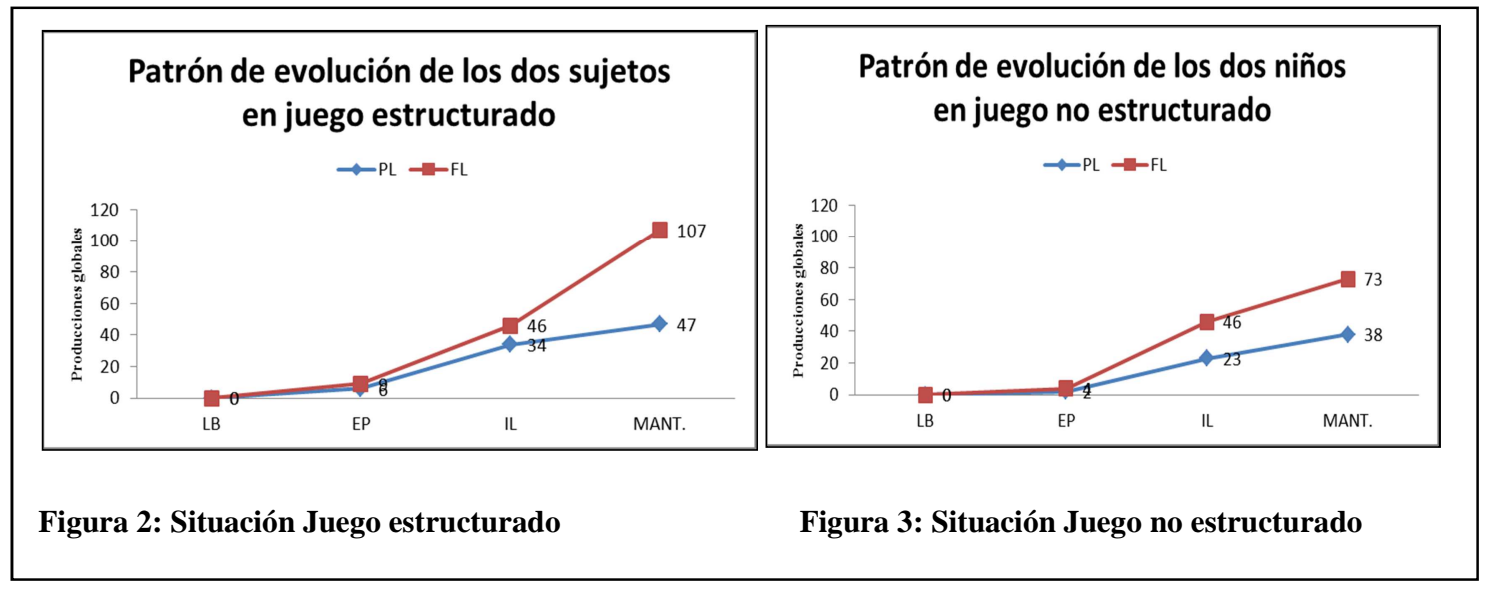

El niño FL obtiene una puntuación de 73 en la situación de juego no estructurado frente a la de 107 en la de juego estructurado. El niño PL no obtiene tan buenos resultados como FL pero aun así pasa de una puntación de 38 a una puntación de 47 elementos. Esta mejoría en el rendimiento en la condición de juego estructurado se puede atribuir claramente a la disposición del método (pasar de una condición de juego no estructurado a una condición de juego estructurado). La accesibilidad de los materiales de forma planificada consigue un aumento mayor de las producciones ya que proporciona una generación directa de los objetivos comunicativos en un entorno predecible y sistemático (Christensen-Sandfort \& Whinnery, 2013). Esta circunstancia la podemos observar si desglosamos los datos por sujeto. Observaremos que la progresión marca estas diferencias aunque con un cierto patrón diferencial: mientras que en el niño FL la diferencia JE vs. JNE es evidente sólo al final del proceso, en el niño PL estas diferencias se evidencias desde las primeras fases de medida. Esto sugiere que un niño (PL) es más sensible al proceso y se beneficia más de la intervención que el otro niño (FL) quien parece disponer de mejores recursos propios y, por tanto, su progreso final es mayor (Ver Figuras 4 y 5). 


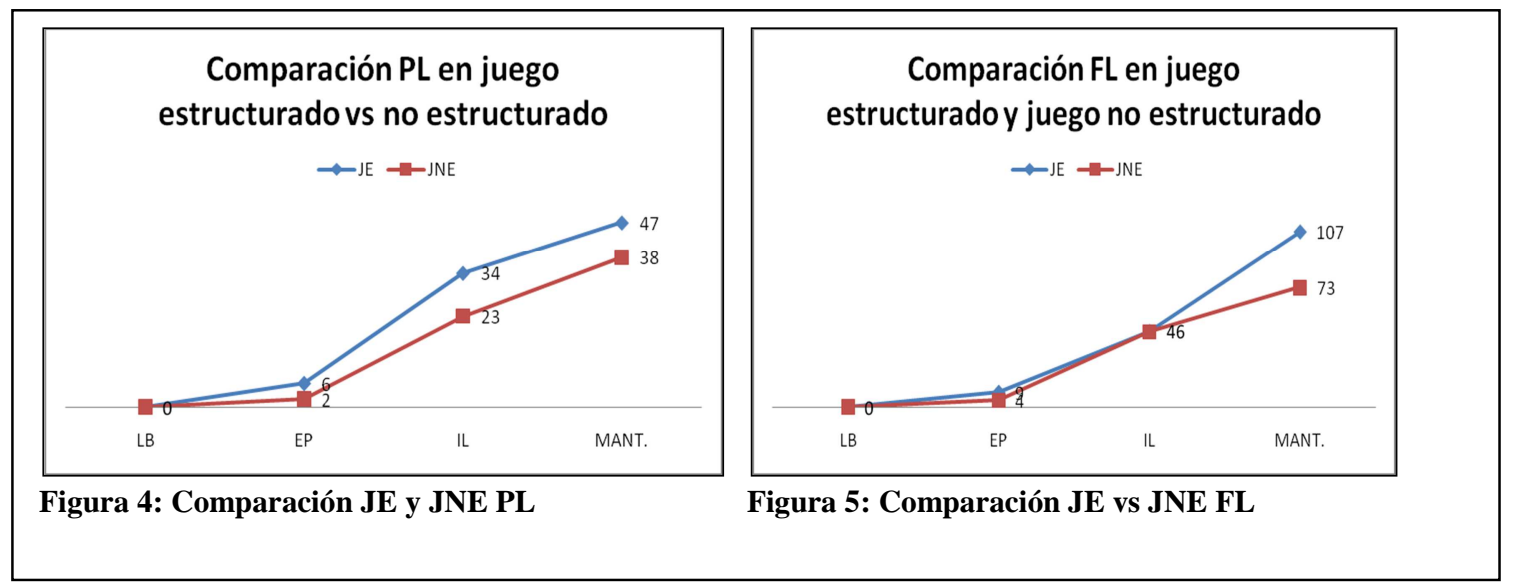

Un tercer aspecto importante a tener en cuenta es la distribución del incremento en las producciones verbales. En ambos niños y en ambas condiciones experimentales (juego estructurado versus juego no estructurado) se registra un bajo incremento en la primera medida (tras el entrenamiento a padres), mientras que se registra un notable incremento en las producciones tras las fase de intervención logopédica. Dado que la evolución es acumulativa, en esta medida podemos observar el resultado tanto del entrenamiento a padres como de la propia fase en la que los niños trabajan exclusivamente con el logopeda en un contexto clínico, mientras los padres prosiguen su trabajo en el hogar. Esta tendencia progresiva se evidencia claramente en el registro tomado tras la fase de mantenimiento en la que el logopeda no interviene directamente siendo los padres los que aplican el método según el entrenamiento recibido. Obviamente, también hemos de considerar que se trata de un efecto acumulativo pues a pesar de que el logopeda no está interviniendo no es posible aislar los efectos del entrenamiento clínico que habían recibido los niños anteriormente. Es nuestra opinión que la intervención del logopeda en el contexto clínico marca un punto de inflexión importante al proporcionar una secuencia estructurada de estimulación que facilita el éxito del niño y el aprendizaje de procedimientos de elicitación y selección de objetivos comunicativos a los padres (complementando la eficacia de los estudios que muestran el entrenamiento a padres Alpert \& Kaiser, 1992; Girolametto, Pearce \& Weitzman, 1997).

En cuarto lugar hemos realizado un análisis comparativo entre las producciones verbales de carácter abierto y las de carácter cerrado. Tal como ya hemos expuesto anteriormente, las producciones verbales de carácter abierto no estaban limitadas 
mientras que las producciones verbales de carácter cerrado tenían un techo numérico. Era importante considerar si se producía un similar incremento o, por el contrario, había diferencias importantes entre ambas clases de producciones.

De acuerdo con los datos (ver las Figuras 6,7, 8 y 9) los resultados muestran que en el caso de las categorías cerradas se produce prácticamente un efecto techo, dado que se alcanzan prácticamente todos los objetivos fijados, mientras que en el caso de las producciones verbales de carácter abierto se produce un notable incremento, igualmente. Este resultado se puede observar tanto en la situación de juego estructurado (JE) como en la situación de juego no estructurado (JNE).

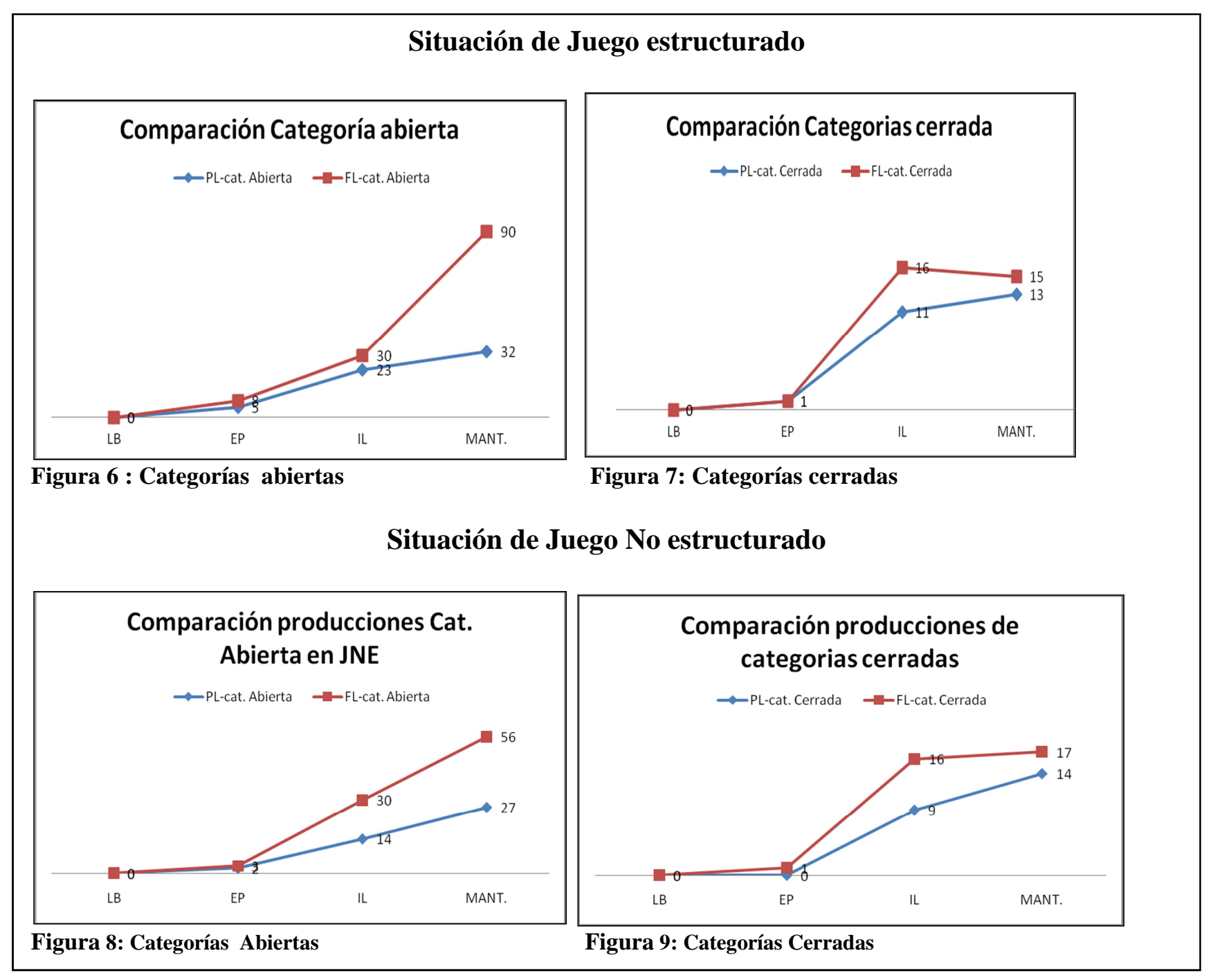


Una observación que podemos realizar en este resultado es que si bien la adquisición de las producciones sigue un patrón progresivo, se puede percibir un patrón diferencial en ambos niños: PL las va adquiriendo continua y paulatinamente mientras que en FL la línea de sus producciones sigue una tendencia de aumento abrupto, a partir de la finalización de la intervención logopédica (Ver Figuras 6, 7, 8 y 9). Esto puede sugerir varias interpretaciones. Por una parte, las producciones de categorías cerradas forman un sustrato que posibilita los intercambios comunicativos y así propicia el avance de las producciones abiertas. Por otra, se confirma que los niños estudiados siguen el mismo patrón evolutivo que los niños normales, en cuanto a las categorías abiertas, sustantivos y acciones. El patrón más típico del léxico inicial es el de que más de la mitad del vocabulario está compuesto por formas nominales, lo cual contribuye al incremento léxico en estos primeros momentos de adquisición. Además los patrones diferenciales encontrados en el incremento del léxico en PL y FL, también responden a los patrones encontrados en el desarrollo léxico inicial de los niños normales, en los que unos siguen un avance paulatino y progresivo mientras que en otros se observa un incremento abrupto (para una revisión más profunda véase Serra i Raventós, 2000).

\section{Discusión}

De los datos anteriores podemos concluir que el método EMTP es un enfoque válido para la intervención logopédica porque muestra ser eficaz en la adquisición de producciones verbales. Esta eficacia se muestra bajo dos premisas importantes: 1) Se puede observar incluso en condiciones poco favorables para la aplicación el método tal como sostenían Hancock y Kaiser (2009). En nuestro trabajo se ha aplicado a dos niños que no reunían los requisitos mínimos fijados por los autores y 2) este método parece incrementar su efectividad si se combina con una intervención de tipo clínico. Es decir, la potencia del enfoque naturalista parece verse incrementada por la inclusión de una fase no naturalista. Se podría, por tanto sostener que un modelo híbrido aporta más eficacia en la intervención que un modelo puramente naturalista aunque esta última afirmación deberá tomarse con prudencia pues dependerá de las circunstancias concretas de cada intervención.

Otra evidencia es que el incremento léxico sigue un patrón evolutivo similar al de los niños de desarrollo normal y las diferencias individuales marcan el desarrollo inicial del léxico. PL, de mayor edad y con un cociente de desarrollo inferior muestra 
una curva de aprendizaje constante y paulatina sugiriendo mayor dependencia del contexto de intervención, tanto clínico como naturalista, para el aprendizaje del lenguaje. Mientras que el cociente de desarrollo de PL, de menor edad y con un rango promedio en su cociente de desarrollo, muestra una curva de aprendizaje abrupta. Esto apoya que las diferencias individuales juegan un papel fundamental en la adquisición del léxico inicial y sugiere que, tanto el mantenimiento de los objetivos comunicativos como su incremento, en los sujetos menos favorecidos requiere continuidad de la intervención y la apropiada selección de los objetivos comunicativos para guiar la actuación de los padres en contexto de intervención natural. Además, el impacto del EMTP sigue el mismo patrón en los dos niños, en la fase de iniciación de las primeras palabras, aun con características diferenciales en edad y cociente de desarrollo que podría relacionarse con diferentes diagnósticos clínicos.

Aún con las limitaciones metodológicas del estudio realizado, los resultados obtenidos confirman nuestro objetivo, es decir, el EMTP favorece el inicio del lenguaje más allá de las condiciones óptimas de aplicación que sugieren Hancock y Kaiser (2009) y por tanto es un método eficaz, también, para aquellos niños que aún no han adquirido las primeras palabras, hito básico para el desarrollo del lenguaje. Estos resultados apoyan la idea de que los predictores del desarrollo del lenguaje siguen el mismo patrón independientemente de la patología (Luyster, Kadlec, Carter \& TagerFlusberg, 2008) y puesto que el inicio tardío de las primeras palabras es un síntoma clínico crítico para el diagnóstico y el pronóstico de los niños con trastornos del desarrollo del lenguaje (Norbury, Tomblin \& Bishop, 2008), el EMTP se muestra como un procedimiento altamente recomendable para uso de los logopedas cuando la intervención persiga el inicio del lenguaje.

En un futuro, se debería volver a evaluar las producciones en ambos participantes, para comprobar su estabilidad en el tiempo así como su generalización a otros contextos y explorar la relación entre los predictores del desarrollo del lenguaje y la eficacia del EMTP.

\section{Referencias}

Aguilar, E. M., y Serrá, M. (2003). AREHA. Análisis del Retraso de Habla. Barcelona: Publicacions y Edicions de la Universitat de Barcelona. 
Alpert, C.L., y Kaiser, A.P. (1992). Training parents as milieu language teachers. Journal of Early Intervention, 16, 31-52.

Baer D. M., Wolf M. M., y Risley T. R. (1968). Some current dimensions of applied behavior analysis. Journal of Applied Behaviour Analisys, 1, 91-97.

Camarata, S. M. (2000). The pragmatics of Paediatric Language Intervention. En N. Müller: Pragmatics in Speech and Language Pathology. Studies in clinical applications (pp. 139-163). Philadelphia: John Benjamin Publishing Company.

Carr, E .G. (1985). Behavioral approaches to language and communication. En E. Schopley \& R. G. B. Mesibov, Communication problems in autism. New York: Plenum Press.

Choi, H. S., y Kim, U. J. (2005). Autism: Using Milieu Teaching Strategies to instruct functional and generalized language. Journal of Special Education: Theory and Practice, 6, 357-375.

Christensen-Sandfort, R. J., y Whinnery, S. B. (2013). Impact of Milieu Teaching on communication skills of young children with autism spectrum disorder. Topics in Early Childhood Special Education 32, 211-222.

Cowan, R.J., y Allen, K. D. (2007). Using naturalistic procedures to enhance learning in individuals with autism: A focus on generalized teaching within the school setting. Psychology in the Schools. Special Issue: Autism Spectrum Disorders, $44,701-715$.

Gilbert, K. (2008). Milieu communication training for late talkers. Perspectives on Language Learning and Education, 15, 112-118.

Girolametto, L., Pearce, P., y Weitzman, E. (1997). Effects of lexical intervention on the phonology of late talkers. Journal of Speech, Language, and Hearing Research, 40, 338-348.

Girolametto, L., Weitzman, E., y Greenberg, J. (2003). Training day care staff to facilitate children's language. American Journal of Speech and Language Pathology, 12, 299-311.

Halle, J. W., Marshall, A. M., y Spradlin, J. E. (1979). Time delay: A technique to increase language use and facilitate generalization in retarded children. Journal of Applied Behavior Analysis, 12, 431-440.

Hancock, T. B., y Kaiser, A. P. (1996). Siblings' use of milieu teaching at home. Topics in Early Childhood Special Education, 16, 168-190. 
Hancock, T. B., y Kaiser, A. P. (2002). The effects of trainer-implemented enhanced milieu teaching on the social communication of children who have autism. Topics in Early Childhood Special Education, 22, 39-54.

Hancock, T. B., y Kaiser, A. P. (2009). Enhanced Milieu Teaching. En R. McCauley \& M. Fey (Eds.), Treatment of Language Disorders in Children (pp. 203-233). Baltimore: Paul Brookes.

Harjusola-Webb, S. M., y Robbins, S. H. (2012). The effects of teacher-implemented naturalistic intervention on the communication of preschoolers with autism. Topics in Early Childhood Special Education, 32, 99-110.

Hart, B., y Rogers-Warren, A. K. (1978). A milieu approach to teaching language. En R. L. Schiefelbusch (Ed.), Bases of language intervention (pp.193-235). Baltimore: University Park Press.

Josse, D. (1997). Escala de desarrollo psicomotor de la primera infancia Brunet-Lezine Revisada. Madrid: Psymtec.

Kaiser, A.P. (1993). Functional language. En M. E. Snell (Ed.), Instruction of students with severe disabilities (pp. 347-379). New York: Macmillan.

Kaiser, A.P., y Delaney, E.M. (2001). Responsive conversations: Creating opportunities for naturalistic language teaching. Young Exceptional Children. Monograph Series, 3, 13-23.

Kaiser, A. P., Hancock, T. B., y Nietfeld, J. P. (2000). The effects of parentimplemented enhanced milieu teaching on the social communication of children who have autism. Journal of Early Education and Development, 4, 423-446.

Kaiser, A. P., Hemmeter, M. L., y Hester, P. P. (1996). The facilitative effect of input on children's language development: Contributions from studies of enhanced milieu teaching. En L. B. Adamson \& M. A. Romski (Eds.), Research on communication and language disorders: Contributions to theories of language development. Baltimore: Paul H. Brookes.

Koegel, R. L., O’Dell, M. C., y Koegel, L. K. (1987). A natural language teaching paradigm for nonverbal autistic children. Journal of Autism and Developmental Disorders, 17, 187-200.

López-Ornat, S., Gallego, C., Gallo, P., Karousou, A., Mariscal, S., y Martínez, M. (2005). MacArthur: Inventario de desarrollo comunicativo. Madrid: TEA Ediciones. 
Luyster, R.J., Kadlec, M.B., Carter, A., y Tager-Flusberg, H. (2008). Language assessment and development in toddlers with autism spectrum disorders. Journal of Autism and Developmental Disorders, 38, 1426-1438.

Manolson, A. (1992). It takes two to talk: A parent's guidebook to helping children Communicate. Toronto: Hanen

McCune-Nicolich, L. (1995). A normative study of representational play at the transition to language. Developmental Psychology, 31, 98-206.

Norbury, C. F., Tomblin, J. B., y Bishop, D. V. M. (2008) Understanding Developmental Language disorders. From theory to practice. N.Y.: Psychology Press.

Serra i Raventó, M., Serrat, E., Solé, R., Bel, A., y Aparici, M. (2000). La adquisición del lenguaje. Barcelona: Ariel.

Sheinkopf, S. J., y Siegel, B. (1998). Home based behavioural treatment of young children with autism. Journal of Autism and Developmental Disorders, 28, 1523.

Smith, T., Groen, A. D., y Wynn, J. W. (2000). Randomized trial of intensive early intervention for children with pervasive developmental disorder. American Journal on Mental Retardation, 105, 269-285.

Vilaseca, R. M., y Del Río, M. J. (1997). La intervención en el área del lenguaje: un modelo interactivo y naturalista. Infancia y Aprendizaje, 77, 3-17.

Warren, S. F., McQuarter, R. J., y Rogers-Warren (1984). The effects of mands and models on the speech of unresponsive socially isolate children. Journal of Speech and Hearing Disorders, 47, 42-52.

Warren, S. F., y Kaiser, A.P. (1986). Generalization of treatment effects by young language- delayed children: A longitudinal analysis. Journal of Speech and Hearing Disorders, 51, 239-251. 